\title{
Shrinkage Estimators of the Reliability Characteristics of a Family of Lifetime Distributions Based on Records
}

\author{
Ajit Chaturvedi and Ananya Malhotra* \\ Department of Statistics, University of Delhi, Delhi - 110007, INDIA \\ *Corresponding Author Email: malhotra.ananya3@gmail.com
}

\begin{abstract}
A family of lifetime distributions is considered which covers many distributions as its special cases. Two measures of reliability are studied, $R(t)=P(X>t)$ and $P=P(X>$ $Y)$. Assuming the availability of some prior information on the parameter of interest, shrinkage estimators are developed for the powers of the scale parameter and reliability functions based on records. These proposed estimators are compared with the maximum likelihood estimators and uniformly minimum variance unbiased estimators of the parametric functions in terms of their relative efficiency. We establish that all the proposed estimators outperform the usual estimators as the true value of parameters approaches their hypothesised value.
\end{abstract}

Keywords and Phrases: Family of lifetime distributions; shrinkage estimation; records; simulation studies

\section{Introduction}

The reliability function $R(t)$ is defined as the probability of failure-free operation until time $t$. Thus, if the random variable $X$ denotes the lifetime of an item or system, then $R(t)=P(X>t)$. Another measure of reliability under stress strength setup is the probability $P=P(X>Y)$ which represents the reliability of an item or system of random strength $X$ subject to random stress $Y$. A lot of work has been done in the literature for the point estimation and testing of $R(t)$ and $P$. Inferences have been drawn for $R(t)$ and $P$ for a family of lifetime distributions by Chaturvedi and Malhotra (2016, 2017).

Many a times, an experimenter may possess some prior knowledge about the experimental conditions based on some past experience of the system under consideration. Thus, he may be able to give an initial guess on the parameter of interest. Given a prior estimate of the parameter, the aim is to obtain an estimator which incorporates this known information. Thompson (1968) introduced the concept of 'shrinkage estimators' wherein a standard estimator can be improved upon, in terms of reduction in its MSE, by shrinking it 
towards its natural origin on multiplying it by a shrinking factor. The idea is to obtain an estimator which is better near the natural origin and possibly worse when farther away. A lot of work has been done in the literature in the direction of shrinkage estimations. Pandey (1983) proposed various shrinkage estimators for the mean of exponential distribution. SiuKeung and Geoffrey (1996), Baklizi (2003) and Baklizi and Abu Dayyeh (2003) proposed shrinkage estimators of $R(t)$ and $P$ for one-parameter exponential distribution.

No one can resist being interested in record values. Records hold value in life-testing experiments. Some examples of record values can be the hottest day ever, the longest winning streak in professional basketball, the lowest stock market figure and so on. Chandler (1952) introduced the concept of record values. Based on records, inferential procedures for the parameters of different distributions have been developed by Habibi et al. (2006), Arashi and Emadi (2008), Razmkhah and Ahmadi (2011), Belaghi et al. (2015) and others. To the best of the knowledge of authors, no inferential procedures are available in literature for the shrinkage estimation of reliability functions based on records.

Let a random variable $(r v) X$ follow a distribution with probability density function $(p d f)$

$$
f(x ; a, \lambda, \underline{\theta})=\frac{G^{\prime}(x ; a, \underline{\theta})}{\lambda} \exp \left(-\frac{G(x ; a, \underline{\theta})}{\lambda}\right) ; x>a \geq 0, \lambda>0
$$

and cumulative distribution function $(c d f)$

$$
F(x ; a, \lambda, \underline{\theta})=1-\exp \left(-\frac{G(x ; a, \underline{\theta})}{\lambda}\right) ; x>a \geq 0, \lambda>0 .
$$

Here, $G(x ; a, \underline{\theta})$ is a function of $x$ and may also depend on the parameters $a$ and $\underline{\theta}$. $\underline{\theta}$ may be vector valued. Moreover, $G(x ; a, \underline{\theta})$ is a monotonically increasing function in $x$ with $G(a ; a, \underline{\theta})=0, G(\infty ; a, \underline{\theta})=\infty$ and $G^{\prime}(x ; a, \underline{\theta})$ denotes the derivative of $G(x ; a, \underline{\theta})$ with respect to $x . \lambda$ is a scale parameter for this family of lifetime distributions.

If we make the transformation $G(x ; a, \underline{\theta})=-\log (1-H(x))$, then

$$
f(x ; \lambda)=\frac{H^{\prime}(x)}{\lambda}[1-H(x)]^{\frac{1}{\lambda}-1}
$$

which is the well-known proportional hazard rate model where $H^{\prime}(x)=\frac{d}{d x} H(x)$. Thus, the proportional hazard rate model considered by Ahmadi et al. (2016) and Basirat et al. (2016) is a particular case of our model in (1.1) and the results of this paper can be extended to the proportional hazard model. 
We note that equation (1.1) represents a family of lifetime distributions since it covers the following lifetime distributions as specific cases:

I. For $G(x ; a, \underline{\theta})=x$ and $a=0$, we get the one-parameter exponential distribution [Johnson and Kotz (1970, p.166)].

II. For $G(x ; a, \underline{\theta})=x^{p}, \underline{\theta}=p, p>0$ and $a=0$, it turns out to be Weibull distribution [Johnson and Kotz (1970, p.250)].

III. For $G(x ; a, \underline{\theta})=x^{2}$ and $a=0$, it gives Rayleigh distribution [Sinha (1986, p. 200)].

IV. For $G(x ; a, \underline{\theta})=\log \left(1+x^{b}\right), \underline{\theta}=b, b>0$, and $a=0$, it leads us to Burr distribution [Burr (1942) and Cislak and Burr (1968)].

V. For $G(x ; a, \underline{\theta})=\log \left(\frac{x}{a}\right)$ and $a>0$, we get Pareto distribution [Johnson and Kotz (1970, p.233)].

VI. For $G(x ; a, \underline{\theta})=\log \left(1+\frac{x}{v}\right), \underline{\theta}=v, v>0$ and $a=0$, it is called Lomax (1954) distribution.

VII. For $G(x ; a, \underline{\theta})=\log \left(1+\frac{x^{b}}{v}\right), \underline{\theta}=(b, v), b>0, v>0$ and $a=0$, it becomes Burr distribution with scale parameter $v$ [Tadikamalla (1980)].

VIII. For $G(x ; a, \underline{\theta})=x^{\gamma} \exp (v x), \underline{\theta}=(\gamma, v), \gamma>0, v>0$ and $a=0$, it gives the modified Weibull distribution of Lai et al. (2003).

IX. For $G(x ; a, \underline{\theta})=(x-a)+\frac{v}{\gamma} \log \left(\frac{x+v}{a+\gamma}\right), \underline{\theta}=(\gamma, v), v>0, \gamma>0$, we $\quad$ get $\quad$ the generalised Pareto distribution of Ljubo (1965).

$\mathrm{X}$. For $G(x ; a, \underline{\theta})=b x+\frac{v}{2} x^{2}, \underline{\theta}=(b, v) v>0, b>0$ and $a=0$, we get the linear exponential distribution [Mahmoud and Al-Nagar (2009)].

XI. For $G(x ; a, \underline{\theta})=\left(1+x^{b}\right)^{v}-1, \underline{\theta}=(b, v), b>0, v>0$ and $a=0$, we get the generalised power Weibull distribution [Nikulin and Haghighi (2006)].

XII. For $G(x ; a, \underline{\theta})=\frac{\beta}{b}\left(e^{b x}-1\right), \underline{\theta}=(b, \beta), \beta>0, b>0$ and $a=0$, we get the Gompertz distribution [Khan and Zia (2009)].

XIII. For $G(x ; a, \underline{\theta})=\left(e^{x^{b}}-1\right), \underline{\theta}=b, b>0$ and $a=0$, this gives Chen (2000) distribution.

XIV. For $G(x ; a, \underline{\theta})=(x-a)$, we get the two-parameter exponential distribution [Ahsanullah (1980)]. 
We note from Chaturvedi and Malhotra (2016) that the reliability function at time $t$ is given by

$$
R(t)=\exp \left(\frac{-G(t ; a, \underline{\theta})}{\lambda}\right)
$$

and for independent rvs $X$ and $Y$ belonging to the same family of distribution, i.e. $G(x ; a, \underline{\theta})=G(y ; a, \underline{\theta})$ with $p d f f\left(x ; a, \lambda_{1}, \underline{\theta}\right)$ and $f\left(y ; a, \lambda_{2}, \underline{\theta}\right)$,

$$
P=\frac{\lambda_{1}}{\lambda_{1}+\lambda_{2}}
$$

Let $X_{1}, X_{2}, \ldots$ be an infinite sequence of independent and identically distributed (iid) rvs from (1.1). An observation $X_{j}$ will be called an upper record value (or simply a record) if its value exceeds than all previous observations. Thus $X_{j}$ is a record if $X_{j}>X_{i}$ for every $i<j$. The record time sequence $\left\{T_{n}, n \geq 0\right\}$ is defined as

$$
\left\{\begin{array}{l}
T_{0}=1 \quad ; \text { with probability } 1 \\
T_{n}=\min \left\{j: X_{j}>X_{T_{n-1}}\right\} ; n \geq 1
\end{array}\right.
$$

and the record value sequence $\left\{R_{n}\right\}$ is then defined as

$$
R_{n}=X_{T_{n}} ; n=0,1,2, \ldots
$$

Assuming the parameters $a$ and $\underline{\theta}$ are known, the likelihood function of the parameter $\lambda$ given the first $n+1$ upper record values $R_{0}, R_{1}, R_{2}, \ldots, R_{n}$ is

$$
L\left(\lambda \mid R_{0}, R_{1}, R_{2}, \ldots, R_{n}\right)=f\left(R_{n} ; a, \lambda, \underline{\theta}\right) \prod_{i=0}^{n-1} \frac{f\left(R_{i} ; a, \lambda, \underline{\theta}\right)}{1-F\left(R_{i} ; a, \lambda, \underline{\theta}\right)} .
$$

It is easy to see that

$$
L\left(\lambda \mid R_{0}, R_{1}, R_{2}, \ldots, R_{n}\right)=\frac{\exp \left(\frac{-G\left(R_{n} ; a, \underline{\theta}\right)}{\lambda}\right)}{\lambda^{n+1}} \prod_{i=0}^{n} G^{\prime}\left(R_{i} ; a, \underline{\theta}\right) .
$$

Chaturvedi and Malhotra (2016) proved using equation (1.5) and the factorization theorem that $G\left(R_{n} ; a, \underline{\theta}\right)$ is a complete and sufficient statistic for $\lambda$ with $p d f$

$$
h\left(G\left(r_{n} ; a, \underline{\theta}\right) \mid \lambda\right)=\frac{G\left(r_{n} ; a, \underline{\theta}\right)^{n}}{\Gamma(n+1) \lambda^{n+1}} \exp \left\{\frac{-G\left(r_{n} ; a, \underline{\theta}\right)}{\lambda}\right\}
$$

and hence they derived the MLES and UMVUES of the parameter $\lambda$ and reliability functions $R(t)$ and $P$.

The purpose of this paper is many-fold. We consider a family of lifetime distributions, which covers as many as fourteen distributions as its specific cases. Assuming the parameters $a$ and $\underline{\theta}$ are known, in Section 2 we propose shrinkage estimators for the powers of the scale parameter $\lambda$. We consider estimation of powers of the parameter because they come in 
expressions for the moments of different distributions and hazard-rate. In Sections 3 and 4, respectively, we develop shrinkage estimators of reliability functions $R(t)$ and $P$. In Section 5 , numerical findings are presented to compare the performance of the proposed shrinkage estimators with the existing MLES and UMVUES. Finally, in Section 6 we discuss the case when all the parameters $\lambda, a$ and $\underline{\theta}$ are unknown and in Section 7 we discuss and conclude our study.

\section{Shrinkage Estimators of Powers of $\lambda$}

Let us suppose that the prior guess value of $\lambda$ is $\lambda_{o}$ and we want to test the hypothesis

$$
\begin{aligned}
& H_{o}: \lambda=\lambda_{o} \\
& H_{1}: \lambda \neq \lambda_{o} .
\end{aligned}
$$

We define the shrinkage estimator of $\lambda^{p}, p \in(-\infty, \infty)$ and $p \neq 0$ based on its MLE as

$$
\widehat{\widehat{\lambda^{p}}}=\alpha_{1} \widehat{\lambda^{p}}+\left(1-\alpha_{1}\right) \lambda_{o}^{p} ; 0 \leq \alpha_{1} \leq 1 \text {, }
$$

where $\alpha_{1}$ is obtained by minimising the MSE of $\widehat{\lambda^{p}}$ and is given by

$$
\alpha_{1}=\frac{\left(\lambda^{p}-\lambda_{o}^{p}\right)\left(E\left(\widehat{\lambda^{p}}\right)-\lambda_{o}^{p}\right)}{E\left(\widehat{\lambda^{2 p}}\right)+\lambda_{o}^{2 p}-2 \lambda_{o}^{p} E\left(\widehat{\lambda^{p}}\right)} .
$$

where,

$$
E\left(\widehat{\lambda^{p}}\right)=\left(\frac{\lambda}{n+1}\right)^{p} \frac{\Gamma(n+1+p)}{\Gamma(n+1)}
$$

and

$$
E\left(\widehat{\lambda^{2 p}}\right)=\left(\frac{\lambda}{n+1}\right)^{2 p} \frac{\Gamma(n+1+2 p)}{\Gamma(n+1)}
$$

Since $\lambda^{p}$ is unknown, we estimate it by its MLE, $\widehat{\lambda^{p}}=\left(\frac{G\left(R_{n} ; a, \underline{\theta}\right)}{n+1}\right)^{p}$. Next, we propose another shrinkage estimator of $\lambda^{p}$ based on its MLE and using the likelihood ratio test. From Chaturvedi and Malhotra (2016), $2 \lambda_{\mathrm{o}}{ }^{-1} G\left(R_{n} ; a, \underline{\theta}\right) \sim \chi_{2(n+1)}^{2}$ and the critical region of the test is given by

$$
\left\{0<G\left(R_{n} ; a, \underline{\theta}\right)<k_{\mathrm{o}}\right\} \cup\left\{k_{\mathrm{o}}{ }^{\prime}<G\left(R_{n} ; a, \underline{\theta}\right)<\infty\right\}
$$

where $k_{\mathrm{o}}$ and $k_{\mathrm{o}}{ }^{\prime}$ are obtained such that $k_{\mathrm{o}}=\frac{\lambda_{\mathrm{o}}}{2} \chi_{2(n+1)}^{2}\left(\frac{\alpha}{2}\right)$ and $k_{\mathrm{o}}{ }^{\prime}=\frac{\lambda_{\mathrm{o}}}{2} \chi_{2(n+1)}^{2}\left(1-\frac{\alpha}{2}\right)$. We state that these critical values are conventional and divide the significance level $\alpha$ equally. Let $\tau_{1}$ be the observed value of $\frac{2 G\left(R_{n} ; a, \underline{\theta}\right)}{\lambda_{o}}$. Then, $p$-value for this test is 


$$
z_{1}=2 \min \left\{1-F\left(\tau_{1}\right), F\left(\tau_{1}\right)\right\}
$$

where $F\left(\tau_{1}\right)$ is the $c d f$ of $\chi^{2}$ distribution with $2(n+1)$ degrees of freedom at the point $\tau_{1}$. Since a large value of $z_{1}$ indicates that $\lambda$ is close to the guess value $\lambda_{o}$ [see Siu-Keung and Geoffrey (1996)], we can use $z_{1}$ to form the shrinkage estimator of $\lambda^{p}$ as

$$
\widehat{\widehat{\lambda_{z_{1}}^{p}}}=\left(1-z_{1}\right) \widehat{\lambda^{p}}+z_{1} \lambda_{o}^{p}
$$

Now, we propose a shrinkage estimator of $\lambda^{p}$ based on its UMVUE as

$$
\widetilde{\lambda^{p}}=\alpha_{2} \widetilde{\lambda^{p}}+\left(1-\alpha_{2}\right) \lambda_{o}^{p} ; 0 \leq \alpha_{2} \leq 1
$$

where $\alpha_{2}$ is obtained by minimising the MSE of $\widetilde{\bar{\lambda}^{p}}$ and is given by

$$
\begin{aligned}
\alpha_{2} & =\frac{\left(\lambda^{p}-\lambda_{o}^{p}\right)\left(E\left(\widetilde{\lambda^{p}}\right)-\lambda_{o}^{p}\right)}{E\left(\widetilde{\lambda^{2 p}}\right)+\lambda_{o}^{2 p}-2 \lambda_{o}^{p} E\left(\widetilde{\lambda^{p}}\right)} \\
& =\frac{\left(\lambda^{p}-\lambda_{o}^{p}\right)^{2}}{\left\{\frac{\Gamma(n+1) \Gamma(n+2 p+1)}{\Gamma^{2}(n+p+1)}\right\} \lambda^{2 p}+\lambda_{o}^{2 p}-2 \lambda_{o}^{p} \lambda^{p}} .
\end{aligned}
$$

Since $\lambda^{p}$ is unknown, we estimate is by its UMVUE, $\widetilde{\lambda^{p}}=\frac{\Gamma(n+1)}{\Gamma(n+p+1)} G\left(R_{n} ; a, \underline{\theta}\right)^{p}$. Also, we can define another shrinkage estimator of $\lambda^{p}$ based on its UMVUE using the $p$-value $z_{1}$ defined above as

$$
\widetilde{\lambda_{z_{1}}^{p}}=\left(1-z_{1}\right) \widetilde{\lambda^{p}}+z_{1} \lambda_{o}^{p}
$$

\section{Shrinkage Estimators of $R(t)$}

Let us suppose that the prior guess value of $R(t)$ is $R_{o}$ and we want to test the hypothesis

$$
\begin{aligned}
& H_{o}: R(t)=R_{o} \\
& H_{1}: R(t) \neq R_{o} .
\end{aligned}
$$

We define the shrinkage estimator of $R(t)$ based on its MLE as

$$
\hat{\hat{R}}(t)=\alpha_{3} \hat{R}(t)+\left(1-\alpha_{3}\right) R_{o} ; 0 \leq \alpha_{3} \leq 1,
$$

where $\alpha_{3}$ is obtained by minimising the MSE of $\hat{R}(t)$ and is given by 


$$
\alpha_{3}=\frac{\left(R(t)-R_{o}\right)\left(E(\hat{R}(t))-R_{o}\right)}{E\left((\hat{R}(t))^{2}\right)+R_{o}^{2}-2 R_{o} E(\hat{R}(t))} .
$$

We get from Chaturvedi and Malhotra (2016) that

$$
E(\hat{R}(t))=\frac{2}{n !}\left[\frac{(n+1) G(t ; a, \underline{\theta})}{\lambda}\right]^{\frac{n+1}{2}} K_{n+1}\left(2 \sqrt{\frac{(n+1) G(t ; a, \underline{\theta})}{\lambda}}\right)
$$

where $K_{r}($.$) is modified Bessel function of second kind of order r$. Similarly,

$$
E\left((\hat{R}(t))^{2}\right)=\frac{2}{n !}\left[2 \frac{(n+1) G(t ; a, \underline{\theta})}{\lambda}\right]^{\frac{n+1}{2}} K_{n+1}\left(2 \sqrt{2 \frac{(n+1) G(t ; a, \underline{\theta})}{\lambda}}\right)
$$

Since $R(t)$ is unknown, we estimate it by its MLE, $\hat{R}(t)=\exp \left(-(n+1) \frac{G(t ; a, \underline{\theta})}{G\left(R_{n} ; a, \underline{\theta}\right)}\right)$. It is worth mentioning here that Baklizi (2003) obtained approximate expressions for $E(\hat{R}(t))$ and $E\left((\hat{R}(t))^{2}\right)$ whereas we have derived their exact expressions. We may note that testing $R(t)=R_{o}$ is equivalent to testing $\lambda=\lambda_{o}$ where $\lambda_{o}=\frac{G(t ; a, \underline{\theta})}{\log \left(\frac{1}{R_{o}}\right)}$. Thus, testing

$$
\begin{aligned}
& H_{o}: R(t)=R_{o} \\
& H_{1}: R(t) \neq R_{o}
\end{aligned}
$$

is equivalent to

$$
\begin{gathered}
H_{o}: \lambda=\lambda_{o} \\
H_{1}: \lambda \neq \lambda_{o}
\end{gathered}
$$

where $\lambda_{o}=\frac{G(t ; a, \underline{\theta})}{\log \left(\frac{1}{R_{o}}\right)}$. On substituting this value of $\lambda_{o}$ in the likelihood ratio test in Section 2, the critical region of the test is given by

$$
\left\{0<G\left(R_{n} ; a, \underline{\theta}\right)<k_{\mathrm{o}}\right\} \cup\left\{k_{\mathrm{o}}{ }^{\prime}<G\left(R_{n} ; a, \underline{\theta}\right)<\infty\right\},
$$

where $k_{\mathrm{o}}$ and $k_{\mathrm{o}}{ }^{\prime}$ are obtained such that

$$
k_{\mathrm{o}}=\frac{G(t ; a, \underline{\theta})}{2 \log \left(\frac{1}{R_{0}}\right)} \chi_{2(n+1)}^{2}\left(\frac{\alpha}{2}\right) \text { and } k_{\mathrm{o}}{ }^{\prime}=\frac{G(t ; a, \underline{\theta})}{2 \log \left(\frac{1}{R_{0}}\right)} \chi_{2(n+1)}^{2}\left(1-\frac{\alpha}{2}\right) .
$$


Let $\tau_{2}$ be the observed value of $\frac{2 G\left(R_{n} ; a, \underline{\theta}\right) \log \left(\frac{1}{R_{o}}\right)}{G(t ; a, \underline{\theta})}$. Then, $p$-value for this test is

$$
z_{2}=2 \min \left\{1-F\left(\tau_{2}\right), F\left(\tau_{2}\right)\right\}
$$

where $F\left(\tau_{2}\right)$ is the $c d f$ of $\chi^{2}$ distribution with $2(n+1)$ degrees of freedom at the point $\tau_{2}$. Since a large value of $z_{2}$ indicates that $R(t)$ is close to the guess value $R_{o}$, we can use $z_{2}$ to form the shrinkage estimator of $R(t)$ as

$$
\hat{R}(t)_{Z_{2}}=\left(1-z_{2}\right) \hat{R}(t)+\left(z_{2}\right) R_{o} .
$$

Now we propose shrinkage estimator of $R(t)$ based on its UMVUE as

$$
\tilde{\tilde{R}}(t)=\alpha_{4} \tilde{R}(t)+\left(1-\alpha_{4}\right) R_{o} ; 0 \leq \alpha_{4} \leq 1,
$$

where $\alpha_{4}$ is obtained by minimising the MSE of $\tilde{\tilde{R}}(t)$ and is given by

$$
\begin{aligned}
\alpha_{4} & =\frac{\left(R(t)-R_{o}\right)\left(E(\tilde{R}(t))-R_{o}\right)}{E\left(\tilde{R}(t)^{2}\right)+R_{o}^{2}-2 R_{o} E(\tilde{R}(t))} \\
& =\frac{\left(R(t)-R_{o}\right)^{2}}{E\left(\tilde{R}(t)^{2}\right)+R_{o}^{2}-2 R_{o} R(t)} .
\end{aligned}
$$

We get from Chaturvedi and Malhotra (2016) that

$$
\begin{aligned}
& E\left(\tilde{R}(t)^{2}\right)=\frac{1}{n !}\left\{\frac{G(t ; a, \underline{\theta})}{\lambda}\right\}^{(n+1)} \exp \left\{\frac{-G(t ; a, \underline{\theta})}{\lambda}\right\}\left[\frac{\lambda a_{n}}{G(t ; a, \underline{\theta})}\right. \\
&-a_{n-1} \exp \left\{\frac{G(t ; a, \underline{\theta})}{\lambda}\right\} E_{i}\left(\frac{-G(t ; a, \underline{\theta})}{\lambda}\right) \\
&+\sum_{i=0}^{n-2} a_{i}\left\{\sum_{m=1}^{n-i-1} \frac{(m-1) !}{(n-i-1) !}\left(\frac{-G(t ; a, \underline{\theta})}{\lambda}\right)^{n-i-m-1}\right. \\
&\left.-\frac{1}{(n-i-1) !}\left(\frac{-G(t ; a, \underline{\theta})}{\lambda}\right)^{n-i-1} \exp \left(\frac{G(t ; a, \underline{\theta})}{\lambda}\right) E_{i}\left(\frac{-G(t ; a, \underline{\theta})}{\lambda}\right)\right\} \\
&\left.+\sum_{i=n+1}^{2 n} a_{i}(i-n) !\left(\frac{\lambda}{G(t ; a, \underline{\theta})}\right)^{i-n+1} \sum_{r=0}^{i-n} \frac{1}{r !}\left(\frac{G(t ; a, \underline{\theta})}{\lambda}\right)^{r}\right]
\end{aligned}
$$

Since $R(t)$ is unknown, we estimate is by its UMVUE, 


$$
\tilde{R}(t)=\left\{\begin{array}{cc}
{\left[1-\frac{G(t ; a, \underline{\theta})}{G\left(R_{n} ; a, \underline{\theta}\right)}\right]^{n} ;} & G(t ; a, \underline{\theta})<G\left(R_{n} ; a, \underline{\theta}\right) . \\
0 \quad & \text { otherwise }
\end{array}\right.
$$

Also, we can define another shrinkage estimator of $R(t)$ based on its UMVUE using $p$-value $z_{2}$ defined above as

$$
\tilde{\tilde{R}}(t)_{z_{2}}=\left(1-z_{2}\right) \tilde{R}(t)+z_{2} R_{o}
$$

\section{Shrinkage Estimators of $P$}

Let us suppose that the prior guess value of $P$ is $P_{o}$ and we want to test the hypothesis

$$
\begin{aligned}
& H_{o}: P=P_{o} \\
& H_{1}: P \neq P_{o} .
\end{aligned}
$$

For $k=\frac{P_{o}}{1-P_{o}}$, this hypothesis is equivalent to

$$
\begin{aligned}
& H_{o}: \lambda_{1}=k \lambda_{2} \\
& H_{1}: \lambda_{1} \neq k \lambda_{2} .
\end{aligned}
$$

We define the shrinkage estimator of $P$ based on its MLE as

$$
\widehat{\hat{P}}=\alpha_{5} \hat{P}+\left(1-\alpha_{5}\right) P_{o} ; 0 \leq \alpha_{5} \leq 1,
$$

where $\alpha_{5}$ is obtained by minimising the MSE of $\hat{\hat{P}}$ and is given by

$$
\alpha_{5}=\frac{\left(P-P_{o}\right)\left(E(\widehat{P})-P_{o}\right)}{E\left(\hat{P}^{2}\right)+P_{o}^{2}-2 P_{o} E(\hat{P})} .
$$

In order to obtain $E(\hat{P})$ and $E\left(\hat{P}^{2}\right)$, we first obtain the $p d f$ of $\hat{P}$. For this we re-write the MLE of $P$ from Chaturvedi and Malhotra (2016) as:

$$
\begin{aligned}
\hat{P} & =\left(1+\frac{\widehat{\lambda_{2}}}{\widehat{\lambda_{1}}}\right)^{-1} \\
& =\left(1+\frac{\lambda_{2}}{\lambda_{1}} F_{(2(m+1), 2(n+1))}\right)^{-1},
\end{aligned}
$$


where the $r v F_{(2(m+1), 2(n+1))}$ follows $F$-distribution with $(2(m+1), 2(n+1))$ degrees of freedom and has $p d f$ :

$$
f(F)=\frac{\left(\frac{m+1}{n+1}\right)^{m+1}}{\beta(m+1, n+1)} \frac{F^{m}}{\left[1+\frac{m+1}{n+1} F\right]^{m+n+2}} ; 0 \leq F \leq \infty .
$$

Making the transformation

$$
\left(1+\frac{\lambda_{2}}{\lambda_{1}} F_{(2(m+1), 2(n+1))}\right)^{-1}=\hat{P}
$$

the $p d f$ of $\hat{P}$ comes out to be

$$
f(\widehat{P})=\frac{\left(\frac{\lambda_{2}}{\lambda_{1}} \frac{(n+1)}{(m+1)}\right)^{n+1}}{\beta(m+1, n+1)} \frac{\hat{P}^{n}(1-\hat{P})^{m}}{\left[1+\hat{P}\left(\frac{\lambda_{2}}{\lambda_{1}} \frac{(n+1)}{(m+1)}-1\right)\right]^{m+n+2}} ; 0 \leq \hat{P} \leq 1
$$

If $\lambda_{1}(m+1)=\lambda_{2}(n+1)$,

$$
f(\hat{P})=\frac{1}{\beta(m+1, n+1)} \hat{P}^{n}(1-\hat{P})^{m} ; 0 \leq \hat{P} \leq 1
$$

and

$$
E\left(\hat{P}^{l}\right)=\frac{\beta(n+l+1, m+1)}{\beta(m+1, n+1)}
$$

If $\lambda_{1}(m+1) \neq \lambda_{2}(n+1)$, then

$$
E\left(\hat{P}^{l}\right)=\frac{\left(\frac{\lambda_{2}}{\lambda_{1}} \frac{(n+1)}{(m+1)}\right)^{n+1}}{\beta(m+1, n+1)} \int_{0}^{1} \frac{\hat{P}^{n+l}(1-\hat{P})^{m}}{\left[1+\hat{P}\left(\frac{\lambda_{2}}{\lambda_{1}} \frac{(n+1)}{(m+1)}-1\right)\right]^{m+n+2}} d \hat{P}
$$

Putting $1+\hat{P}\left(\frac{\lambda_{2}}{\lambda_{1}} \frac{(n+1)}{(m+1)}-1\right)=u$,

$$
E\left(\hat{P}^{l}\right)=\frac{\left(\frac{\lambda_{2}}{\lambda_{1}} \frac{(n+1)}{\beta(m+1)}\right)^{n+1}}{\beta(m+1, n+1)}\left(\frac{\lambda_{2}}{\lambda_{1}} \frac{(n+1)}{(m+1)}-1\right)^{-l-n-m-1} \int_{1}^{\frac{\lambda_{2}(n+1)}{\lambda_{1}(m+1)}} u^{-n-m-2}(u-1)^{l+n}\left(\frac{\lambda_{2}}{\lambda_{1}} \frac{(n+1)}{(m+1)}-u\right)^{m} d u
$$




$$
\begin{aligned}
= & \frac{1}{\beta(m+1, n+1)}\left(\frac{\lambda_{1}(m+1)}{\lambda_{2}(n+1)}\right)^{l}\left[1-\frac{\lambda_{1}(m+1)}{\lambda_{2}(n+1)}\right]^{-l-m-n-1} \sum_{j=0}^{m}(-1)^{j}\left(\begin{array}{c}
m \\
j
\end{array}\right)\left(\frac{\lambda_{1}(m+1)}{\lambda_{2}(n+1)}\right)^{j} \\
& \sum_{k=0}^{n+l}(-1)^{k}\left(\begin{array}{c}
n+l \\
k
\end{array}\right) I\left(\frac{\lambda_{2}}{\lambda_{1}} \frac{(n+1)}{(m+1)}, j+k-n-m-2\right),
\end{aligned}
$$

where $I(c, p)=\int_{1}^{c} t^{p} d t=\left\{\begin{array}{l}\frac{c^{p+1}-1}{p+1} ; p \neq-1 \\ \log (c) ; p=-1\end{array}\right.$.

Since $\lambda_{1}$ and $\lambda_{2}$ are unknown, we estimate them by their MLES. Next, we propose another shrinkage estimator of $P$ based on the MLE and using the likelihood ratio test. From Chaturvedi and Malhotra (2016),

$$
\frac{G\left(R_{n} ; a, \underline{\theta}\right)}{G\left(R_{m}^{*} ; a, \underline{\theta}\right)} \sim \frac{(n+1) \lambda_{1}}{(m+1) \lambda_{2}} F_{2(n+1), 2(m+1)},
$$

and the critical region is given by

$$
\left\{\frac{G\left(R_{n} ; a, \underline{\theta}\right)}{G\left(R_{m}^{*} ; a, \underline{\theta}\right)}<k_{2}\right\} \cup\left\{\frac{G\left(R_{n} ; a, \underline{\theta}\right)}{G\left(R_{m}^{*} ; a, \underline{\theta}\right)}>k_{2}{ }^{\prime}\right\},
$$

where $k_{2}=\frac{k(n+1)}{(m+1)} F_{2(n+1), 2(m+1)}\left(\frac{\alpha}{2}\right)$ and $k_{2}{ }^{\prime}=\frac{k(n+1)}{(m+1)} F_{2(n+1), 2(m+1)}\left(1-\frac{\alpha}{2}\right)$. We state that these critical values are conventional and divide the significance level $\alpha$ equally.Let $\tau_{3}$ be the observed value of $\left(\frac{G\left(R_{n} ; a, \underline{\theta}\right)}{k G\left(R_{m}^{*} ; a, \underline{\theta}\right)}\right)\left(\frac{m+1}{n+1}\right)$. Then, $p-$ value for this test is

$$
z_{3}=2 \min \left\{1-F\left(\tau_{3}\right), F\left(\tau_{3}\right)\right\}
$$

where $F\left(\tau_{3}\right)$ is the $c d f$ of $F$-distribution with $(2(n+1), 2(m+1))$ degrees of freedom at the point $\tau_{3}$. Since a large value of $z_{3}$ indicates that $P$ is close to the guess value $P_{o}$, we can use $z_{3}$ to form the shrinkage estimator of $P$ as

$$
\hat{\hat{P}}_{z_{3}}=\left(1-z_{3}\right) \hat{P}+z_{3} P_{o}
$$

Now, we propose a shrinkage estimator of $P$ based on UMVUE as

$$
\tilde{\widetilde{P}}=\alpha_{6} \tilde{P}+\left(1-\alpha_{6}\right) P_{o} ; 0 \leq \alpha_{6} \leq 1,
$$

where $\alpha_{6}$ is obtained by minimising the MSE of $\tilde{\widetilde{P}}$ and is given by 


$$
\begin{aligned}
\alpha_{6} & =\frac{\left(P-P_{o}\right)\left(E(\tilde{P})-P_{o}\right)}{E\left(\tilde{P}^{2}\right)+P_{o}^{2}-2 P_{o} E(\tilde{P})} \\
& =\frac{\left(P-P_{o}\right)^{2}}{E\left(\tilde{P}^{2}\right)+P_{o}^{2}-2 P_{o} P} .
\end{aligned}
$$

To obtain the expression for $E\left(\tilde{P}^{2}\right)$, from Chaturvedi and Malhotra (2016) we get

$$
\begin{aligned}
E\left(\tilde{P}^{2}\right)= & \sum_{i=0}^{m-1} \sum_{j=0}^{m-1} a_{i} a_{j} E\left[\left(\frac{G\left(R_{n} ; a_{1}, \underline{\theta_{1}}\right)}{G\left(R_{m} ; a_{2}, \underline{\theta_{2}}\right)}\right)^{i+j+2} I\left(G\left(R_{n} ; a_{1}, \underline{\theta_{1}}\right)<G\left(R_{m} ; a_{2}, \underline{\theta_{2}}\right)\right)\right] \\
& +\sum_{i=0}^{n-1} \sum_{j=0}^{n-1} b_{i} b_{j} E\left[\left(\frac{G\left(R_{m} ; a_{2}, \underline{\theta}_{2}\right)}{G\left(R_{n} ; a_{1}, \underline{\theta_{1}}\right)}\right)^{i+j} I\left(G\left(R_{m} ; a_{2}, \underline{\theta_{2}}\right)<G\left(R_{n} ; a_{1}, \underline{\theta_{1}}\right)\right)\right]
\end{aligned}
$$

where $a_{i}=\frac{(-1)^{i} m ! n !}{(m-1-i) !(n+i+1) !}$ and $b_{i}=\frac{(-1)^{i} m ! n !}{(m+i) !(n-i) !}$. We have for a $r v \quad F$ following $F$-distribution with $(2(n+1), 2(m+1))$ degrees of freedom,

$$
\begin{aligned}
& E\left[\left(\frac{G\left(R_{n} ; a_{1}, \underline{\theta_{1}}\right)}{G\left(R_{m} ; a_{2}, \underline{\theta_{2}}\right)}\right)^{i+j+2} I\left(G\left(R_{n} ; a_{1}, \underline{\theta_{1}}\right)<G\left(R_{m} ; a_{2}, \underline{\theta_{2}}\right)\right)\right] \\
& =\frac{\left(\frac{\lambda_{1}}{\lambda_{2}}\right)^{i+j+2}\left(\frac{n+1}{m+1}\right)^{i+j+n+3}}{\beta(n+1, m+1)} \int_{0}^{1} \frac{F^{n+i+j+2}}{\left[1+\left(\frac{n+1}{m+1}\right) F\right]^{n+m+2}} d F .
\end{aligned}
$$

Similarly,

$$
\begin{gathered}
E\left[\left(\frac{G\left(R_{m} ; a_{2}, \underline{\theta}_{2}\right)}{G\left(R_{n} ; a_{1}, \underline{\theta_{1}}\right)}\right)^{i+j} I\left(G\left(R_{m} ; a_{2}, \underline{\theta}_{2}\right)<G\left(R_{n} ; a_{1}, \underline{\theta_{1}}\right)\right)\right] \\
=\frac{\left(\frac{\lambda_{2}}{\lambda_{1}}\right)^{i+j}\left(\frac{m+1}{n+1}\right)^{i+j+m+1}}{\beta(m+1, n+1)} \int_{0}^{1} \frac{F^{m+i+j}}{\left[1+\left(\frac{m+1}{n+1}\right) F\right]^{n+m+2}} d F .
\end{gathered}
$$

Since $\lambda_{1}$ and $\lambda_{2}$ are unknown, they are estimated by their UMVUES. Finally, we propose a shrinkage estimator of $P$ based on its UMVUE using the $p-$ value $z_{3}$ defined above as 


$$
\tilde{\tilde{P}}_{z_{3}}=\left(1-z_{3}\right) \tilde{P}+z_{3} P_{o}
$$

\section{Numerical Findings}

In this section we investigate the performance of the proposed estimators in previous sections through Monte Carlo simulation. First, we compare the shrinkage estimators of $\lambda^{p}$ with its MLE, $\widehat{\lambda^{p}}$ and UMVUE, $\widetilde{\lambda^{p}}$. Since the family of lifetime distributions in (1.1) has fourteen distributions as special cases, thus in order to obtain generalised results using the distribution property of records in equation (1.6), we simulate random numbers from the distribution of the complete and sufficient statistic of the family (1.1) for a specified number of records $n+1$ and scale parameter $\lambda$ [see Arnold et al. (1998)]. In this regard, we simulate 1000 random numbers from gamma distribution with shape parameter $n+1=5$ and scale parameter $\lambda$ where $\lambda$ is sequence from $1,2, \ldots, 15$. For each value of $\lambda$, we calculate the average estimate of the MLE and UMVUE of $\lambda^{p}$ which in turn are used to calculate the $\alpha_{1}$ and $\alpha_{2}$ respectively. Using these values we calculate the MSE of proposed shrinkage estimators of $\lambda^{p}$. The results are shown in Table 1 .

Table 1: Shrinkage estimators of $\lambda$ when the hypothesised value of $\lambda$ is $\lambda_{o}=3$.

\begin{tabular}{ccccccc}
\hline$\lambda$ & $\hat{\lambda}$ & $\tilde{\lambda}$ & $\hat{\hat{\lambda}}$ & $\hat{\hat{\lambda}}_{z_{1}}$ & $\tilde{\tilde{\lambda}}$ & $\tilde{\tilde{\lambda}}_{z_{1}}$ \\
\hline 2.000 & 2.017543 & 2.017543 & 2.800457 & 2.450565 & 2.423054 & 2.450565 \\
2.500 & 2.521929 & 2.521929 & 2.985296 & 2.896483 & 2.915205 & 2.896483 \\
2.995 & 3.021270 & 3.021270 & 3.000001 & 3.002600 & 3.000006 & 3.002600 \\
2.996 & 3.022279 & 3.022279 & 3.000001 & 3.002738 & 3.000007 & 3.002738 \\
2.997 & 3.023288 & 3.023288 & 3.000001 & 3.002877 & 3.000008 & 3.002877 \\
2.998 & 3.024297 & 3.024297 & 3.000001 & 3.003017 & 3.000009 & 3.003017 \\
2.999 & 3.025306 & 3.025306 & 3.000002 & 3.003159 & 3.000011 & 3.003159 \\
3.000 & 3.026314 & 3.026314 & 3.000002 & 3.003302 & 3.000012 & 3.003302 \\
3.001 & 3.027323 & 3.027323 & 3.000002 & 3.003446 & 3.000013 & 3.003446 \\
3.002 & 3.028332 & 3.028332 & 3.000002 & 3.003591 & 3.000015 & 3.003591 \\
3.003 & 3.029341 & 3.029341 & 3.000002 & 3.003738 & 3.000017 & 3.003738 \\
3.004 & 3.030349 & 3.030349 & 3.000003 & 3.003886 & 3.000018 & 3.003886 \\
3.005 & 3.031358 & 3.031358 & 3.000003 & 3.004035 & 3.000020 & 3.004035 \\
3.500 & 3.530700 & 3.530700 & 3.010248 & 3.219778 & 3.063353 & 3.219778 \\
4.000 & 4.035086 & 4.035086 & 3.058176 & 3.652327 & 3.292993 & 3.652327 \\
\hline
\end{tabular}


We observe from Table 1 that when the true value of parameter $\lambda$ is close to $\lambda_{o}$, the shrinkage estimators are more accurate than the MLES and UMVUES but are less accurate when $\lambda$ is far away from $\lambda_{o}$.

Suppose the prior guess value of $\lambda$ is $\lambda_{o}=4.5$. In other words, we want to test the hypothesis

$$
\begin{aligned}
& H_{o}: \lambda=4.5 \\
& H_{1}: \lambda \neq 4.5 .
\end{aligned}
$$

Let us define $\phi=\frac{\lambda}{\lambda_{o}}$. Table 2 shows the relative efficiencies of the shrinkage estimators with respect to the usual MLES and UMVUES of $\lambda^{p}$ for different values of $\lambda$ and its powers $p$. These are obtained by the following formula:

$$
R E\left(\vartheta, \vartheta_{S E}\right)=\frac{\operatorname{MSE}(\vartheta)}{\operatorname{MSE}\left(\vartheta_{S E}\right)}
$$

where $\vartheta$ is the MLE (UMVUE) of the parameter and $\vartheta_{S E}$ is the shrinkage estimator of the parameter based on MLE (UMVUE).

\begin{tabular}{|c|c|c|c|c|c|c|c|c|c|c|c|c|}
\hline \multirow[b]{2}{*}{$\phi$} & \multicolumn{4}{|c|}{$p=-1$} & \multicolumn{4}{|c|}{$p=1$} & \multicolumn{4}{|c|}{$p=2$} \\
\hline & $\hat{\hat{\lambda}}$ & $\hat{\hat{\lambda}}_{z_{1}}$ & $\tilde{\tilde{\lambda}}$ & $\tilde{\tilde{\lambda}}_{z_{1}}$ & $\hat{\hat{\lambda}}$ & $\hat{\hat{\lambda}}_{z_{1}}$ & $\tilde{\tilde{\lambda}}$ & $\tilde{\tilde{\lambda}}_{z_{1}}$ & $\hat{\hat{\lambda}}$ & $\hat{\hat{\lambda}}_{z_{1}}$ & $\tilde{\tilde{\lambda}}$ & $\tilde{\tilde{\lambda}}_{z_{1}}$ \\
\hline 0.22 & 1.728 & 0.974 & 1.355 & 0.961 & 0.977 & 0.984 & 0.986 & 0.984 & 0.990 & 0.954 & 0.997 & 0.988 \\
\hline 0.44 & 2.319 & 1.287 & 1.707 & 1.182 & 0.977 & 1.066 & 1.070 & 1.066 & 0.990 & 0.865 & 1.030 & 0.908 \\
\hline 0.67 & 4.753 & 3.341 & 2.814 & 2.397 & 0.996 & 1.601 & 1.610 & 1.601 & 1.349 & 1.360 & 1.378 & 1.325 \\
\hline 0.89 & 35.324 & 39.113 & 20.929 & 19.733 & 10.880 & 11.452 & 11.508 & 11.452 & 14.227 & 13.138 & 8.969 & 10.959 \\
\hline 1.11 & 32.400 & 6.738 & 7.613 & 7.505 & 17.003 & 7.490 & 17.812 & 7.490 & 30.648 & 9.271 & 20.077 & 7.859 \\
\hline 1.33 & 3.673 & 1.895 & 2.230 & 2.101 & 2.976 & 2.214 & 3.712 & 2.214 & 6.843 & 2.570 & 4.688 & 2.326 \\
\hline 1.56 & 1.486 & 1.250 & 1.487 & 1.356 & 1.631 & 1.429 & 2.331 & 1.429 & 4.386 & 1.541 & 3.101 & 1.475 \\
\hline 1.78 & 0.957 & 1.063 & 1.247 & 1.119 & 1.218 & 1.164 & 1.887 & 1.164 & 3.593 & 1.210 & 2.567 & 1.191 \\
\hline 2.00 & 0.839 & 0.997 & 1.139 & 1.023 & 1.039 & 1.056 & 1.678 & 1.056 & 3.225 & 1.081 & 2.310 & 1.077 \\
\hline 2.22 & 0.929 & 0.972 & 1.081 & 0.982 & 0.948 & 1.009 & 1.559 & 1.009 & 3.018 & 1.027 & 2.164 & 1.030 \\
\hline 2.44 & 0.960 & 0.964 & 1.047 & 0.964 & 0.899 & 0.990 & 1.483 & 0.990 & 2.886 & 1.005 & 2.070 & 1.010 \\
\hline 2.67 & 0.960 & 0.961 & 1.025 & 0.956 & 0.873 & 0.982 & 1.431 & 0.982 & 2.795 & 0.995 & 2.006 & 1.002 \\
\hline 2.89 & 0.960 & 0.960 & 1.009 & 0.953 & 0.859 & 0.979 & 1.393 & 0.979 & 2.728 & 0.992 & 1.961 & 0.999 \\
\hline 3.11 & 0.960 & 0.960 & 0.998 & 0.952 & 0.853 & 0.978 & 1.364 & 0.978 & 2.677 & 0.990 & 1.926 & 0.997 \\
\hline 3.33 & 0.960 & 0.960 & 0.990 & 0.952 & 0.853 & 0.977 & 1.342 & 0.977 & 2.635 & 0.990 & 1.900 & 0.997 \\
\hline
\end{tabular}

Table 2: Relative Efficiency of Shrinkage estimators of $\lambda^{p}$ with respect to MLE and UMVUE of $\lambda^{p}$ for $n+1=5, \lambda \in(1,2, \ldots, 15)$ and $\lambda_{o}=4.5$. 
We observe from Table 2 that when $\phi$ is close to 1 , i.e. as $\lambda$ approaches $\lambda_{o}$, all the proposed shrinkage estimators of $\lambda^{p}$ have relative efficiency greater than 1 . This fact can also be illustrated in the following Figure 1. We see that all the shrinkage estimators are highly efficient in the neighbourhood of $\phi=1$, i.e. when the true value of $\lambda$ is close to the hypothesised value $\lambda_{o}$.

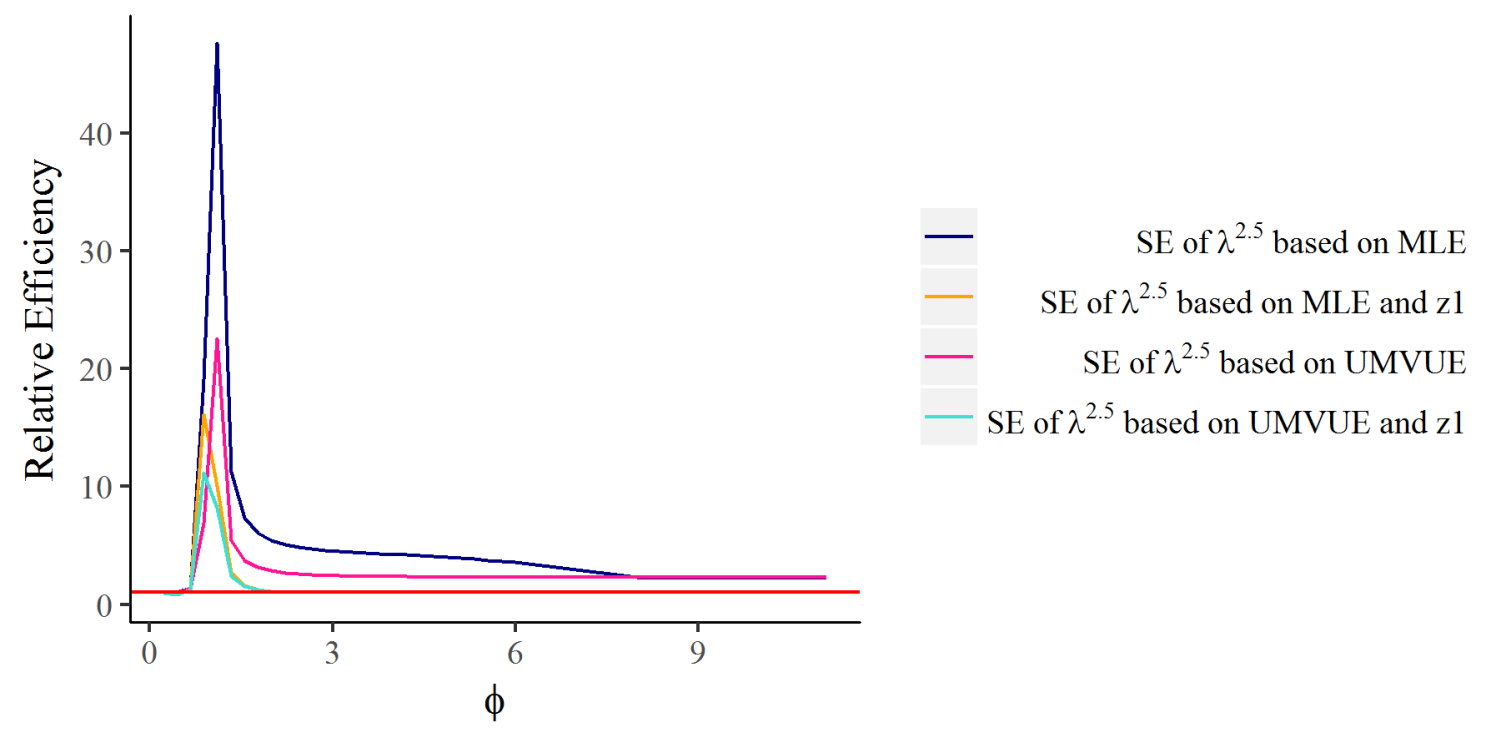

Figure 1: Relative Efficiency of Shrinkage estimators of $\lambda^{2.5}$ with respect to MLE and UMVUE of $\lambda^{2.5}$.

Now, on similar lines we compare the performance of shrinkage estimators of $R(t)$ with its MLE, $\hat{R}(t)$ and UMVUE, $\tilde{R}(t)$ [Chaturvedi and Malhotra (2016)]. For several values of $\lambda$, a specified time $t=2$ and number of records $n+1=5$, we calculate the average estimate of MLE and UMVUE of $R(t)$. Using these values we compute $\alpha_{3}$ and $\alpha_{4}$ and hence obtain the shrinkage estimators of $R(t)$. Table 3 shows the estimators of $R(t)$ developed in (3.1), (3.3), (3.4) and (3.6).

Table 3: Shrinkage estimators of $R(t)$ when the hypothesised value of $R(t)$ is $R_{o}=0.7$.

\begin{tabular}{ccccccc}
\hline$R(t)$ & $\hat{R}(t)$ & $\tilde{R}(t)$ & $\widehat{\hat{R}}(t)$ & $\widehat{\hat{R}}(t)_{z_{2}}$ & $\widetilde{\widetilde{R}}(t)$ & $\widetilde{\widetilde{R}}(t)_{z_{2}}$ \\
\hline 0.200 & 0.202819 & 0.213184 & 0.236487 & 0.205404 & 0.700000 & 0.215716 \\
0.400 & 0.403200 & 0.440096 & 0.474326 & 0.422841 & 0.700000 & 0.457296 \\
0.695 & 0.697202 & 0.733462 & 0.699980 & 0.699756 & 0.700000 & 0.702915 \\
0.696 & 0.698197 & 0.734389 & 0.699992 & 0.699829 & 0.700000 & 0.703258 \\
0.697 & 0.699191 & 0.735316 & 0.699998 & 0.699917 & 0.700000 & 0.703617 \\
0.698 & 0.700186 & 0.736243 & 0.700000 & 0.700020 & 0.700000 & 0.703989 \\
0.699 & 0.701180 & 0.737169 & 0.700000 & 0.700139 & 0.700000 & 0.704377 \\
0.700 & 0.702174 & 0.738095 & 0.700000 & 0.700273 & 0.700000 & 0.704780 \\
0.701 & 0.703169 & 0.739020 & 0.700000 & 0.700422 & 0.700000 & 0.705198
\end{tabular}




\begin{tabular}{lllllll}
0.702 & 0.704163 & 0.739945 & 0.700000 & 0.700587 & 0.700000 & 0.705630 \\
0.703 & 0.705157 & 0.740870 & 0.700000 & 0.700767 & 0.700000 & 0.706078 \\
0.704 & 0.706152 & 0.741794 & 0.700000 & 0.700963 & 0.700000 & 0.706540 \\
0.705 & 0.707146 & 0.742718 & 0.700000 & 0.701174 & 0.700000 & 0.707018 \\
0.800 & 0.801554 & 0.828764 & 0.755687 & 0.785215 & 0.700033 & 0.808048 \\
0.900 & 0.900825 & 0.915941 & 0.900825 & 0.900805 & 0.915941 & 0.915920 \\
\hline
\end{tabular}

We observe from Table 3 that when the true value of parameter $R(t)$ is close to $R_{o}$, the shrinkage estimators are more accurate than the MLES and UMVUES but are less accurate when $R(t)$ is far away from $R_{o}$.

Suppose the prior guess value of $R(t)$ is $R_{o}=0.7$, i.e. we want to test the hypothesis

$$
\begin{aligned}
& H_{o}: R(t)=0.7 \\
& H_{1}: R(t) \neq 0.7 .
\end{aligned}
$$

As explained in Section 3, this is similar to testing

$$
\begin{aligned}
& H_{o}: \lambda=\lambda_{o} \\
& H_{1}: \lambda \neq \lambda_{o}
\end{aligned}
$$

and we proceed as above. In Table 4 we show the relative efficiencies of shrinkage estimators of $R(t)$ with respect to its MLE and UMVUE for several values of $\lambda$. In particular we display

\begin{tabular}{|c|c|c|c|c|c|c|c|c|c|}
\hline \multicolumn{6}{|c|}{$\lambda=(1,2, \ldots, 20), \lambda_{o}=5.6, G(t ; a, \underline{\theta})=t$} & \multicolumn{4}{|c|}{$\lambda=(6,7, \ldots, 25), \lambda_{o}=11.21, G(t ; a, \underline{\theta})=t^{2}$} \\
\hline$\phi$ & $\widehat{\hat{R}}(t)$ & $\widehat{\hat{R}}(t)_{z_{2}}$ & $\widetilde{\widetilde{R}}(t)$ & $\widetilde{\widetilde{R}}(t)_{z_{2}}$ & $\phi$ & $\hat{\hat{R}}(t)$ & $\widehat{\hat{R}}(t)_{Z_{2}}$ & $\widetilde{\widetilde{R}}(t)$ & $\widetilde{\widetilde{R}}(t)_{z_{2}}$ \\
\hline 0.18 & 0.989 & 0.975 & 0.039 & 0.584 & 0.54 & 2.056 & 1.678 & 0.644 & 1.422 \\
\hline 0.36 & 1.345 & 1.111 & 0.220 & 1.064 & 0.62 & 2.771 & 2.363 & 1.115 & 1.852 \\
\hline 0.54 & 2.056 & 1.678 & 0.644 & 1.422 & 0.71 & 4.191 & 3.769 & 2.106 & 2.751 \\
\hline 0.71 & 4.191 & 3.769 & 2.106 & 2.751 & 0.80 & 7.845 & 7.469 & 4.778 & 5.207 \\
\hline 0.89 & 23.670 & 23.675 & 16.863 & 17.173 & 0.89 & 23.670 & 23.675 & 16.863 & 17.173 \\
\hline 1.07 & 51.348 & 10.871 & 44.187 & 12.632 & 0.98 & 699.629 & 133.775 & 567.156 & 104.388 \\
\hline 1.25 & 4.683 & 2.485 & 3.752 & 2.884 & 1.07 & 51.348 & 10.871 & 44.192 & 12.632 \\
\hline 1.43 & 2.073 & 1.492 & 1.337 & 1.682 & 1.16 & 10.497 & 4.138 & 8.861 & 4.845 \\
\hline 1.61 & 1.435 & 1.190 & 0.692 & 1.296 & 1.25 & 4.683 & 2.485 & 3.755 & 2.884 \\
\hline 1.78 & 1.197 & 1.068 & 0.426 & 1.126 & 1.34 & 2.860 & 1.824 & 2.089 & 2.089 \\
\hline 1.96 & 1.088 & 1.014 & 0.291 & 1.043 & 1.43 & 2.073 & 1.492 & 1.340 & 1.682 \\
\hline 2.14 & 1.032 & 0.988 & 0.212 & 1.000 & 1.52 & 1.668 & 1.305 & 0.938 & 1.446 \\
\hline 2.32 & 1.001 & 0.977 & 0.162 & 0.977 & 1.61 & 1.435 & 1.190 & 0.697 & 1.296 \\
\hline 2.50 & 0.984 & 0.971 & 0.128 & 0.966 & 1.69 & 1.291 & 1.117 & 0.541 & 1.196 \\
\hline 2.68 & 0.974 & 0.969 & 0.105 & 0.960 & 1.78 & 1.197 & 1.068 & 0.435 & 1.126 \\
\hline
\end{tabular}
results for records from one-parameter exponential distribution and Rayleigh distribution.

Table 4: Relative Efficiency of Shrinkage estimators of $R(t)$ with respect to MLE and UMVUE of $R(t)$ for $n+1=5$. 


\begin{tabular}{l|llll|l|llll}
2.85 & 0.969 & 0.967 & 0.088 & 0.957 & 1.87 & 1.133 & 1.036 & 0.361 & 1.078 \\
3.03 & 0.966 & 0.967 & 0.076 & 0.956 & 1.96 & 1.088 & 1.014 & 0.308 & 1.043 \\
3.21 & 0.966 & 0.966 & 0.069 & 0.955 & 2.05 & 1.056 & 0.999 & 0.277 & 1.018 \\
3.39 & 0.966 & 0.966 & 0.072 & 0.954 & 2.14 & 1.032 & 0.988 & 0.287 & 1.000 \\
3.57 & 0.965 & 0.965 & 0.954 & 0.954 & 2.23 & 1.014 & 0.981 & 0.183 & 0.987 \\
\hline
\end{tabular}

We observe from Table 4 that when $\phi$ is close to 1 , i.e. as $R(t)$ approaches $R_{o}$, all the proposed shrinkage estimators of $R(t)$ have relative efficiency greater than 1 . This fact can also be illustrated in the following Figure 2. We see that all the shrinkage estimators are highly efficient in the neighbourhood of $\phi=1$, i.e. when the true value of $R(t)$ is close to the hypothesised value $R_{o}$.

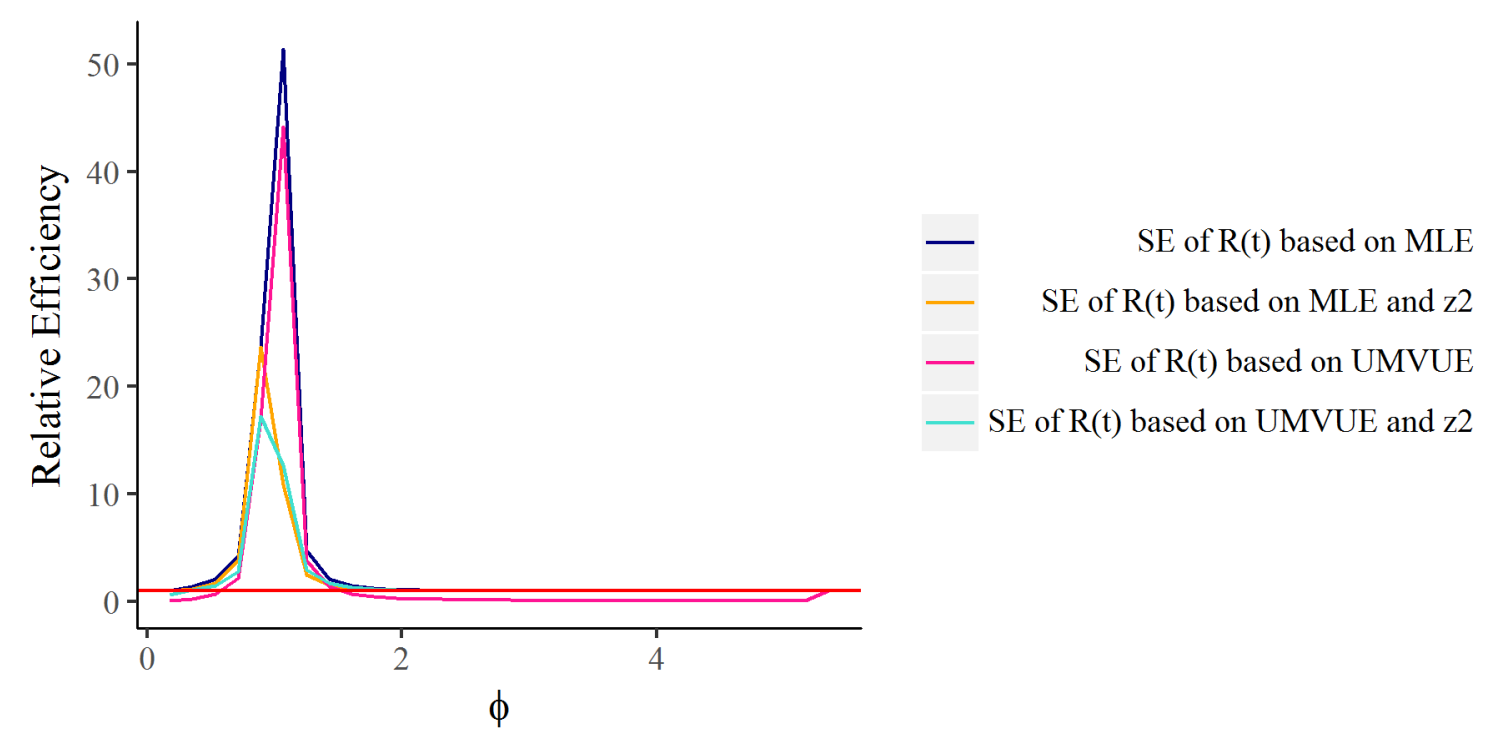

Figure 2: Relative Efficiency of Shrinkage estimators of $R(t)$ with respect to MLE and UMVUE of $R(t)$.

Next, we compare the performance of shrinkage estimators of $P$ with its MLE, $\widehat{P}$ and UMVUE, $\tilde{P}$ [Chaturvedi and Malhotra (2016)]. Let $X$ and $Y$ be two independent $r v s$ from the same family of distributions and with scale parameter $\lambda_{1}$ and $\lambda_{2}$ respectively. For fixed number of records $(n+1$ and $m+1)$ and fixed scale parameters, we generate 1000 random numbers each from the distribution of the complete and sufficient statistic of $\lambda_{1}$ and $\lambda_{2}$. Using (1.4), we compute $P(X>Y)$ and calculate the average estimate of MLE and UMVUE of $P$. Using these values we compute $\alpha_{5}$ and $\alpha_{6}$ and hence obtain the shrinkage estimators of $P$. Table 5 shows the various estimators of $P$ developed in equations (4.1), (4.3), (4.4) and (4.6).

Table 5: Shrinkage estimators of $P$ when the hypothesised value of $P$ is $P=0.8$. 


\begin{tabular}{ccccccc}
\hline$P$ & $\hat{P}$ & $\tilde{P}$ & $\widehat{\hat{P}}$ & $\hat{\widehat{P}}_{Z_{3}}$ & $\widetilde{\widetilde{P}}$ & $\widetilde{\tilde{P}}_{Z_{3}}$ \\
\hline 0.600 & 0.602970 & 0.628312 & 0.678909 & 0.637763 & 0.628312 & 0.658630 \\
0.700 & 0.702596 & 0.735551 & 0.765819 & 0.747674 & 0.800000 & 0.765378 \\
0.795 & 0.797012 & 0.828839 & 0.799981 & 0.799963 & 0.800000 & 0.800358 \\
0.796 & 0.798005 & 0.829773 & 0.799992 & 0.799960 & 0.800000 & 0.800590 \\
0.797 & 0.798997 & 0.830706 & 0.799998 & 0.799973 & 0.800000 & 0.800837 \\
0.798 & 0.799990 & 0.831638 & 0.800000 & 0.800000 & 0.800000 & 0.801098 \\
0.799 & 0.800983 & 0.832569 & 0.800000 & 0.800041 & 0.800000 & 0.801374 \\
0.800 & 0.801975 & 0.833499 & 0.800000 & 0.800098 & 0.800000 & 0.801665 \\
0.801 & 0.802968 & 0.834428 & 0.800000 & 0.800170 & 0.800000 & 0.801970 \\
0.802 & 0.803960 & 0.835356 & 0.800000 & 0.800257 & 0.800000 & 0.802291 \\
0.803 & 0.804953 & 0.836283 & 0.800000 & 0.800359 & 0.800000 & 0.802626 \\
0.804 & 0.805945 & 0.837209 & 0.800000 & 0.800476 & 0.800000 & 0.802977 \\
0.805 & 0.806938 & 0.838134 & 0.800000 & 0.800608 & 0.800000 & 0.803342 \\
0.850 & 0.851573 & 0.878723 & 0.806843 & 0.823061 & 0.800000 & 0.835201 \\
0.900 & 0.901110 & 0.921484 & 0.868841 & 0.881681 & 0.800000 & 0.898140 \\
\hline
\end{tabular}

We observe from Table 5 that when the true value of parameter $P$ is close to $P_{o}$, the shrinkage estimators are more accurate than the MLES and UMVUES but are less accurate when $P$ is far away from $P_{o}$.

Suppose the prior guess value of $P$ is $P_{o}=0.8$, i.e. we want to test the hypothesis

$$
\begin{aligned}
& H_{o}: P=P_{o} \\
& H_{1}: P \neq P_{o} .
\end{aligned}
$$

As explained in Section 4, this is equivalent to testing

$$
\begin{aligned}
& H_{o}: \lambda_{1}=k \lambda_{2} \\
& H_{1}: \lambda_{1} \neq k \lambda_{2} .
\end{aligned}
$$

We define $\phi=\frac{\lambda_{1}}{k \lambda_{2}}$. In Table 6 we show the relative efficiencies of shrinkage estimators of $P$ with respect to its MLE and UMVUE for several values of $\lambda_{1}$.

Table 6: Relative Efficiency of Shrinkage estimators of $P$ with respect to MLE and UMVUE of $P$ based on records. $\lambda_{1}$ is sequence from 10 to $40, \lambda_{2}=7, n+1=3$ and $m+1=5$.

\begin{tabular}{c|cccc}
\hline$\phi$ & $\hat{\hat{P}}$ & $\widehat{\hat{P}}_{Z_{3}}$ & $\widetilde{\widetilde{P}}$ & $\widetilde{\widetilde{P}}_{Z_{3}}$ \\
\hline 0.36 & 0.409 & 0.373 & 0.348 & 0.381 \\
0.39 & 0.499 & 0.448 & 0.395 & 0.449 \\
0.43 & 0.607 & 0.538 & 0.445 & 0.529 \\
0.46 & 0.740 & 0.646 & 1.093 & 0.624 \\
0.50 & 0.905 & 0.778 & 1.330 & 0.740 \\
0.54 & 1.114 & 0.944 & 1.625 & 0.882
\end{tabular}




\begin{tabular}{lcccc}
0.57 & 1.384 & 1.156 & 2.000 & 1.063 \\
0.61 & 1.739 & 1.431 & 2.484 & 1.297 \\
0.64 & 2.218 & 1.800 & 3.127 & 1.609 \\
0.68 & 2.885 & 2.310 & 4.003 & 2.037 \\
0.71 & 3.847 & 3.041 & 5.238 & 2.649 \\
0.75 & 5.295 & 4.140 & 7.056 & 3.567 \\
0.79 & 7.601 & 5.898 & 9.885 & 5.033 \\
0.82 & 11.556 & 8.958 & 14.620 & 7.583 \\
0.86 & 19.093 & 14.969 & 23.423 & 12.614 \\
0.89 & 35.975 & 29.274 & 42.628 & 24.731 \\
0.93 & 86.184 & 77.483 & 98.046 & 67.103 \\
0.96 & 372.883 & 384.559 & 400.381 & 358.514 \\
1.00 & Inf & 554.555 & Inf & 470.029 \\
1.04 & 432.829 & 151.667 & 415.851 & 172.286 \\
1.07 & 111.049 & 60.148 & 105.789 & 73.236 \\
1.11 & 50.593 & 31.659 & 47.799 & 39.175 \\
1.14 & 29.141 & 19.535 & 27.311 & 24.225 \\
1.18 & 19.078 & 13.303 & 17.741 & 16.453 \\
1.21 & 13.823 & 9.681 & 12.496 & 11.918 \\
1.25 & 10.557 & 7.390 & 9.305 & 9.047 \\
1.29 & 8.366 & 5.846 & 7.216 & 7.115 \\
1.32 & 6.811 & 4.755 & 5.772 & 5.752 \\
1.36 & 5.661 & 3.955 & 4.730 & 4.754 \\
1.39 & 4.781 & 3.349 & 3.954 & 4.001 \\
1.43 & 4.090 & 2.879 & 3.358 & 3.419 \\
\hline & & & &
\end{tabular}

We observe from Table 6 that when $\phi$ is close to 1 , i.e. as $P$ approaches $P_{o}$, all the proposed shrinkage estimators of $P$ have relative efficiency greater than 1 . This fact can also be illustrated in the following Figure 3. We see that all the shrinkage estimators are highly efficient in the neighbourhood of $\phi=1$, i.e. when the true value of $P$ is close to the hypothesised value $P_{o}$ or when $\lambda_{1}$ is close to $P_{o}\left(1-P_{o}\right)^{-1} \lambda_{2}$. 


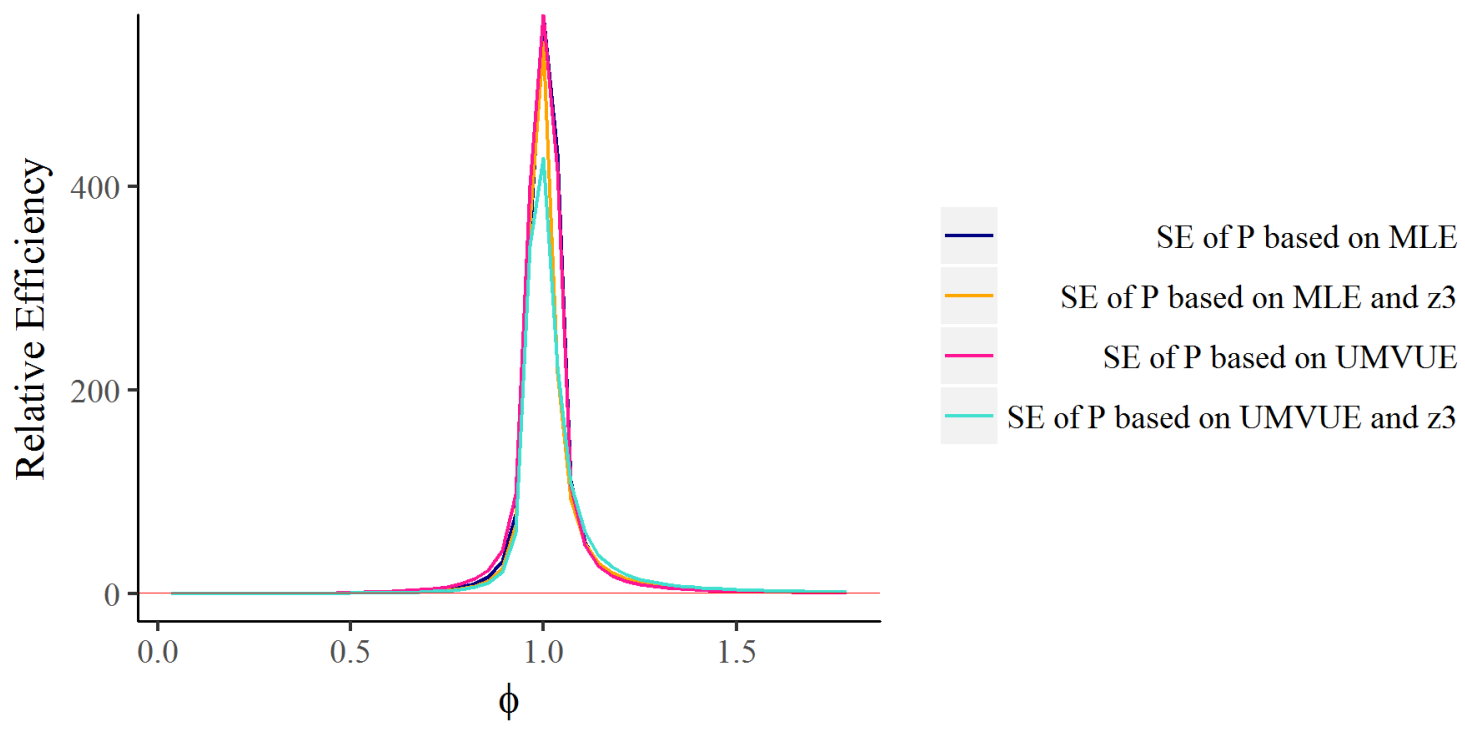

Figure 3: Relative Efficiency of Shrinkage estimators of $P$ with respect to MLE and UMVUE of $P$.

\section{Shrinkage Estimators of $\lambda, R(t)$ and $P$ when all the Parameters are Unknown}

In this section we discuss the case when all the parameters of the family of lifetime distributions in (1.1) are unknown. Thus, the log-likelihood equation of the parameters $\lambda, a$ and $\underline{\theta}$ given the $n+1$ upper record values $R_{0}, R_{1}, \ldots, R_{n}$ is

$$
l\left(\lambda, a, \underline{\theta} \mid R_{0}, R_{1}, \ldots, R_{n}\right)=-(n+1) \log (\lambda)-\frac{G\left(R_{n} ; a, \underline{\theta}\right)}{\lambda}+\sum_{i=0}^{n} \log \left(G^{\prime}\left(R_{i} ; a, \underline{\theta}\right)\right)
$$

Since the derivative of the log-likelihood function with respect to the parameters $\lambda, a$ and $\underline{\theta}$ respectively does not have a simultaneous closed form solution, we proceed with our discussion through an illustrative example. We consider the case of Weibull distribution by taking $G(x ; a, \underline{\theta})=x^{p}, a=0$ and $\underline{\theta}=p$. The MLES of the unknown parameters $\lambda$ and $p$ are the solutions of the following two simultaneous equations:

$$
\frac{-(n+1)}{\lambda}+\frac{R_{n}^{p}}{\lambda^{2}}=0
$$

and

$$
\frac{-R_{n}^{p}}{\lambda} \log \left(R_{n}\right)+\sum_{i=0}^{n} \frac{p \log \left(R_{i}\right)+1}{p}=0
$$


Since these non-linear equations don't have a closed form solution, therefore we apply Newton Raphson algorithm to obtain the MLES of $\lambda$ and $p$ as $\hat{\lambda}$ and $\hat{p}$ respectively.

Now we propose shrinkage estimators of powers of $\lambda, R(t)$ and $P$ based on their respective MLES using the likelihood ratio test.

For testing $H_{o}: \lambda=\lambda_{o}$ against $H_{1}: \lambda \neq \lambda_{o}$, the shrinkage estimator of $\lambda^{q}, q \in(-\infty, \infty)$ and $q \neq 0$, based on $p-$ value $z_{1}$ is defined as

$$
\widehat{\lambda_{z_{1}}^{q}}=\left(1-z_{1}\right) \widehat{\lambda^{q}}+z_{1} \lambda_{o}^{q}
$$

where $\widehat{\lambda^{q}}=\left(\frac{R_{n}^{\hat{p}}}{n+1}\right)^{q}$ is the MLE of $\lambda^{q}$ with $\hat{p}$ obtained as the MLE of $p$ from NewtonRaphson algorithm. For $\tau_{1}=\frac{2 R_{n}^{\widehat{p}}}{\lambda_{o}}$,

$$
z_{1}=2 \min \left\{1-F\left(\tau_{1}\right), F\left(\tau_{1}\right)\right\},
$$

where $F\left(\tau_{1}\right)$ is the $c d f$ of $\chi^{2}$ distribution with $2(n+1)$ degrees of freedom at the point $\tau_{1}$.

Next, for testing $H_{o}: R(t)=R_{o}$ against $H_{1}: R(t) \neq R_{o}$, the shrinkage estimator of $R(t)$ based on $p-$ value $z_{2}$ is defined as

$$
\hat{\hat{R}}(t)_{Z_{2}}=\left(1-z_{2}\right) \hat{R}(t)+\left(z_{2}\right) R_{o}
$$

where $\hat{R}(t)=\exp \left(\frac{-(n+1) t \hat{p}}{R_{n}^{\hat{p}}}\right)$ the MLE of $R(t)$ obtained by invariance property of MLE. For $\tau_{2}=\frac{2 R_{n}^{\hat{p}} \log \left(\frac{1}{R_{0}}\right)}{t^{\hat{p}}}$

$$
z_{2}=2 \min \left\{1-F\left(\tau_{2}\right), F\left(\tau_{2}\right)\right\}
$$

where $F\left(\tau_{2}\right)$ is the $c d f$ of $\chi^{2}$ distribution with $2(n+1)$ degrees of freedom at the point $\tau_{2}$.

Finally, consider the independent rvs $X$ and $Y$ belonging to the same family of distributions, say Weibull distribution with $p d f f\left(x ; \lambda_{1}, p_{1}\right)$ and $f\left(y ; \lambda_{2}, p_{2}\right)$. Let $R_{0}, R_{1}, \ldots, R_{n}$ and $R_{0}^{*}, R_{1}^{*}, \ldots, R_{m}^{*}$ be the record value sequences from the distribution of $X$ and $Y$ respectively. Then, for testing $H_{o}: P=P_{o}$ against $H_{1}: P \neq P_{o}$, the shrinkage estimator of $P$ based on $p$-value $z_{3}$ is defined as 


$$
\widehat{\hat{P}}_{z_{3}}=\left(1-z_{3}\right) \hat{P}+z_{3} P_{o}
$$

where $\widehat{P}=\frac{\widehat{\lambda_{1}}}{\widehat{\lambda_{1}}+\widehat{\lambda_{2}}}$ is the MLE of $P$ with $\widehat{\lambda_{1}}=\frac{R_{n}^{\widehat{p_{1}}}}{n+1}$ and $\widehat{\lambda_{2}}=\frac{R_{m}^{*} \widehat{p_{2}}}{m+1}$. For $\tau_{3}=\frac{R_{n}^{\widehat{p_{1}}}}{k R_{m}^{*} \widehat{p_{2}}}\left(\frac{m+1}{n+1}\right)$,

$$
z_{3}=2 \min \left\{1-F\left(\tau_{3}\right), F\left(\tau_{3}\right)\right\}
$$

where $F\left(\tau_{3}\right)$ is the $c d f$ of $F$-distribution with $(2(n+1), 2(m+1))$ degrees of freedom at the point $\tau_{3}$.

The procedure of obtaining shrinkage estimators of parametric functions discussed above can be easily generalised for any model of this family for which all the model parameters are unknown. The log-likelihood equation can be iteratively solved for MLES of the unknown parameters and using some prior information on the parameters, shrinkage estimators can easily be developed. In order to avoid repetition of the above procedure, we restrict ourselves to one example on Weibull distribution having more than one unknown parameters.

Note that the UMVUES of $\lambda^{q}, R(t)$ and $P$ do not exist under this case and hence we cannot define their respect shrinkage estimators when all the parameters are unknown.

\section{An Example}

Let us simulate 1000 random numbers each from the distribution of $X \sim$ Weibull $\left(\lambda_{1}=\right.$ $\left.2, p_{1}=2\right)$ and $Y \sim \operatorname{Weibull}\left(\lambda_{2}=5, p_{2}=1\right)$. Then based on $n+1=7$ record values from the distribution of $X$ and $m+1=10$ record values from the distribution of $Y$, the MLES of the parameters $\lambda_{1}, p_{1}, \lambda_{2}$ and $p_{2}$ obtained from Newton Raphson algorithm are $\widehat{\lambda_{1}}=2.9517, \widehat{p_{1}}=$ $1.4400, \widehat{\lambda_{2}}=4.9274$ and $\widehat{p_{2}}=1.2194$ respectively. The MLE of $R(t)$ based on sample $X$ is $\hat{R}(t)=0.5650$ and the MLE of $P=P(X>Y)$ is $\hat{P}=0.3746$.

Now, suppose for sample $X$, the prior guess value of $\lambda_{1}$ is $\lambda_{o}=3$. Then the shrinkage estimator of $\lambda_{1}$ based on $p$-value $z_{1}=0.1500$ is $\widehat{\overline{\lambda_{1}}}=2.9590$. Similarly, if the prior guess value of $R(t)$ based on sample $X$ is $R_{o}=0.4$, then the shrinkage estimator of $R(t)$ based on $p$-value $z_{2}=0.1500$ is $\hat{\hat{R}}(t)=0.5409$. Finally, if the prior guess value of $P$ is $P_{o}=0.4$, then the shrinkage estimator of $P$ based on $p-$ value $z_{3}=0.1552$ is $\hat{P}=0.3785$.

\section{Discussion}


In this paper we proposed several shrinkage estimators of powers of the scale parameter $\lambda$ and reliability functions $R(t)$ and $P$ of the family of lifetime distributions in (1.1) under the assumption that parameters $a$ and $\underline{\theta}$ are known. This study is of particular interest in cases where we have record data and some prior knowledge of the parameters of our model. We conducted extensive simulation studies in Section 5 to investigate the performance of these shrinkage estimators in comparison to the MLES and UMVUES based on records. On the basis of relative efficiencies computed for these shrinkage estimators, we can conclude that all of the proposed estimators of the parametric functions are way more efficient than the usual MLES and UMVUES based on record values in the neighbourhood of the null its hypothesis, i.e. when the true value of the parameter close to its prior guess value. Thus, we were able to establish improved estimators of various parametric functions. In Section 6, we developed a technique of obtaining shrinkage estimators of parametric functions in the case when all the parameters of the family of distributions are unknown.

\section{Acknowledgement}

We are grateful to the Editor in Chief, the Associate Editors and the Reviewers for their fruitful suggestions which lead to a considerable improvement in this research paper.

\section{References}

1. Ahmadi, J., Mirfarah, E. and Parsian, A. (2016): Comparison of preliminary test estimators based on generalized order statistics from proportional hazard family using Pitman measure of closeness. Commun. Statist. - Theo. Meth., DOI:10.1080/03610926.2015.1060336.

2. Ahsanullah, M. (1980): Linear prediction of record values for the two parameter exponential distribution. Ann. Inst. Statist. Math., 32, Part A, 363-368.

3. Arnold, B. C., Balakrishnan, N. and Nagaraja, H. N. (1998): Record. John Wiley and Sons, New York.

4. Arashi, M. and Emadi, M. (2008): Evidential inference based on record data and inter-record times. Stat. Papers, 13(8), 380-210.

5. Baklizi, A. (2003): Shrinkage estimation of the exponential reliability with censored data. Focus on Applied Statistics, 195-204.

6. Baklizi, A. and Dayyeh, W. A. (2003): Shrinkage estimation of $\mathrm{P}(\mathrm{Y}<\mathrm{X})$ in the exponential case. Commun. Statist. - Simul., 32(1), 31-42. 
7. Basirat, M., Baratpour, S. and Ahmadi, J. (2016): On estimation of stress-strength parameter using record values from proportional hazard rate models. Commun. Statist. - Theo. Meth., DOI: 10.1080/03610926.2014.948727.

8. Belaghi, R. A., Arashi, M. and Tabatabaey, S. M. M. (2015): On the Construction of Preliminary Test Estimator Based on Record Values for the Burr XII Model. Commun. Statist. - Theo. Meth., 44(1), 1-23, DOI:10.1080/03610926.2012.733473.

9. Burr, I. W (1942): Cumulative Frequency Functions. Ann. Math. Statist., 13 (2), 215232.

10. Burr, I. W. and Cislak, P. J. (1968): On a general system of distributions: I. Its curveshaped characteristics; II. The sample median, Jour. Amer. Statist. Assoc., 63, 627635.

11. Chandler, K. N. (1952): The distribution and frequency of record values. Jour. Royal Statist. Socie., Series B, 14, 220-228.

12. Chaturvedi, A. and Malhotra, A. (2016): Estimation and Testing Procedures for the Reliability Functions of a Family of Lifetime Distributions based on Records, Int. J. Syst. Assur. Eng. Manag., DOI:10.1007/s13198-016-0531-2.

13. Chaturvedi, A. and Malhotra, A. (2017): Inference on the Parameters and Reliability Characteristics of three parameter Burr Distribution based on Records. Appl. Math. Inf. Scien., 11(3), 837-849.

14. Chen, Z. (2000): A new two- parameter lifetime distribution with bathtub shape or increasing failure rate function. Statist. Prob. Letters, 49, 155-161.

15. Habibi, R. A., Arghami, N. R. and Ahmadi, J. (2006): Statistical evidence in experiments and in record values. Commun. Stat.- Theo. Meth. 35(11), 1971-1983.

16. Johnson, N. L. and Kotz, S. (1970): Distributions in Statistics-Continuous Univariate Distributons, Vol. 1, New York: John Wiley \& Sons, Vol. 1- 2.

17. Khan, R. U. and Zia, B. (2009): Recurrence Relations for single and Product moments of record values from Gompertz distribution and a characterization. World App. Sci. Jour., 7(10), 1331-1334.

18. Lai, C. D., Xie, M. and Murthy, D. N. P. (2003): A Modified Weibull model. IEEE Trans. Reliability, 52, 33-37.

19. Ljubo, M. (1965): Curves and concentration indices for certain generalized Pareto distributions. Statist. Rev., 15, 257-260. 
20. Lomax, K. S. (1954): Business failures. Another example of the analysis of failure data. Jour. Amer. Statist. Assoc., 49, 847-852.

21. Nikulin, M. and Haghighi, F. (2006): A chi-squared test for the generalized power weibull family for the head-and-neck cancer censored data. Jour. Math. Sci., 133(3), 1333- 1341.

22. Pandey, B. (1983): Shrinkage estimation of the exponential scale parameter. IEEE Trans. Reliability, 2, 203-205.

23. Razmkhah, M. and Ahmadi, J. (2011): Comparing two sampling schemes based on entropy of record statistics. Statist. Papers, 53, 95-106.

24. Sinha, S. K. (1986): Reliability and Life Testing. Wiley Eastern Limited, New Delhi.

25. Siu-Keung,T. and Geoffrey, T. (1996): Shrinkage estimation of reliability for exponentially distributed lifetimes. Commun. Statist.-Simul., 25(2), 415-430.

26. Tadikamalla, P. R. (1980): A look at the Burr and related distributions. Inter. Statist. Rev., 48, 337-344.

27. Thompson, J. R. (1968): Some shrinkage techniques for estimating the mean. Jour. Amer. Statist. Assoc., 63(321), 113-122. 